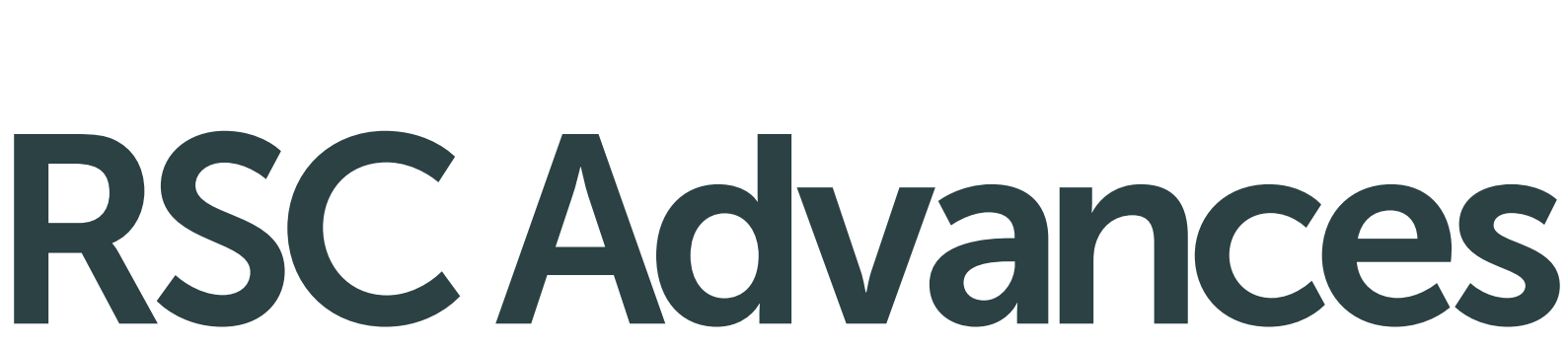

This article can be cited before page numbers have been issued, to do this please use: M. Bououdina, A. Dakhel, :. M. El-Hilo, D. H. Anjum, M. B. Kanoun and S. Goumri-Said, RSC Adv., 2015, DOI:

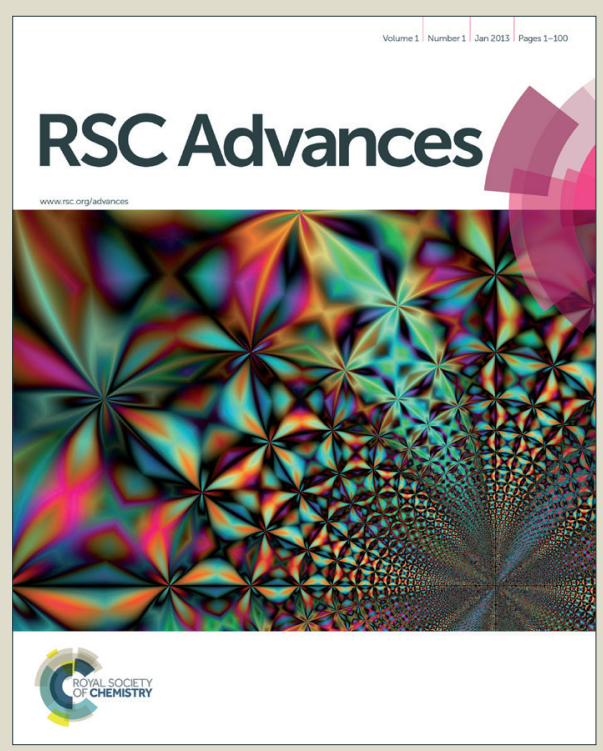

This is an Accepted Manuscript, which has been through the Royal Society of Chemistry peer review process and has been accepted for publication.

Accepted Manuscripts are published online shortly after acceptance, before technical editing, formatting and proof reading. Using this free service, authors can make their results available to the community, in citable form, before we publish the edited article. This Accepted Manuscript will be replaced by the edited, formatted and paginated article as soon as this is available.

You can find more information about Accepted Manuscripts in the Information for Authors.

Please note that technical editing may introduce minor changes to the text and/or graphics, which may alter content. The journal's standard Terms \& Conditions and the Ethical guidelines still apply. In no event shall the Royal Society of Chemistry be held responsible for any errors or omissions in this Accepted Manuscript or any consequences arising from the use of any information it contains. 


\title{
Revealing a room temperature ferromagnetism in cadmium oxide nanoparticles: An experimental and first-principles study
}

\author{
M. Bououdina ${ }^{1,2, *}$, A. A. Dakhel ${ }^{1}$, M. El-Hilo ${ }^{1}$, D. H. Anjum ${ }^{3}$, M. B. Kanoun ${ }^{4}$ \\ and S. Goumri-Said ${ }^{5, \dagger}$ \\ ${ }^{1}$ Department of Physics, College of Science, University of Bahrain, P.O. Box 32038, Kingdom of Bahrain \\ ${ }^{2}$ Nanotechnology Centre, University of Bahrain, P.O. Box 32038, Kingdom of Bahrain \\ ${ }^{3}$ Advanced Nanofabrication and Imaging Core Lab, King Abdullah University of Science and \\ Technology, Thuwal, Saudi Arabia \\ ${ }^{4}$ School of Physics, Georgia Institute of Technology Atlanta, Georgia 30332-0400, USA \\ ${ }^{5}$ School of Chemistry and Biochemistry and Center for Organic Photonics and Electronics \\ Georgia Institute of Technology Atlanta, Georgia 30332-0400, USA
}

Email : Souraya.goumri-said@chemistry.gatech.edu

\begin{abstract}
We obtain a single cadmium oxide phase from powder synthesized by thermal decomposition method of cadmium acetate dehydrate. The yielded powder is annealed in air, vacuum, and $\mathrm{H}_{2}$ gas in order to create point defects. Magnetization-field curves reveal the appearance of diamagnetic behavior with a ferromagnetic component for all the powders. Powder annealing under vacuum $\left(\mathrm{H}_{2}\right.$ atmosphere $(\sim 1.2 \mathrm{memu} / \mathrm{g})$ leads to a saturation magnetization increases by $\sim 3.6 \%(\sim 9 \%)$ leading to oxygen vacancies (Cd vacancies) respectively. The magnetic analysis shows a room temperature ferromagnetic (RTFM) component together with known diamagnetic properties. The appearance of RTFM takes origin from oxygen and cadmium vacancies. ab-initio calculations performed on the $\mathrm{CdO}$ nanoparticles show that the magnetism is governed by polarized hybrid states of the $\mathrm{Cd} d$ and $\mathrm{O} p$ orbitals together with the vacancy.
\end{abstract}

PACS : 78.67.Bf, 74.62.Dh, 75.47.Lx, 31.15.ej 


\section{Introduction}

Cadmium oxide $(\mathrm{CdO})$ is among one of transparent conducting oxides (TCOs) with a resistivity in the range $\sim 10^{-2}-10^{-4} \Omega \mathrm{cm}$ and good transparency in Vis and NIR spectral region. It has a direct bandgap within the range $~ 2.2-2.7 \mathrm{eV}$ [1-3] Owing to its superior physical properties; $\mathrm{CdO}$ was used in many optoelectronic applications like solar cells, smart windows, transparent conducting oxides etc [1-4] The electrical conduction properties in TCOs including $\mathrm{CdO}$ are known to be due to oxygen vacancies $\left(\mathrm{V}_{\mathrm{O}}\right)$ and cadmium interstitials $\left(\mathrm{Cd}_{\mathrm{i}}\right)$. Therefore, it is possible to tailor its optoelectronic properties by controlling those point defects. Many possible methods are used to modify the density of point defects including annealing processes under different reactive gases or vacuum was well as by doping with foreign ions.

Traditionally, considerable attention has been focused on dilute magnetic semiconductors and diluted magnetic oxides, in which local magnetic moments are introduced by doping elements with partially $3 d$ or $4 f$ subshells [5]. However, unexpected ferromagnetism (FM) has been observed in $\mathrm{HfO}_{2}$ thin films without any doping [6]. This type of " $d^{0}$ ferromagnetism" [7] provides a challenge to understand the origin of the magnetism. From experimental and theoretical studies, it seems that the FM is attributed to oxygen or Hf vacancies [8]. Moreover, theoretical calculations have suggested that the FM can be induced by cation vacancies in $\mathrm{TiO}_{2}$, $\mathrm{ZnO}$, and $\mathrm{SnO}_{2}$ [9-12], or by anion vacancies in $\mathrm{CeO}_{2}$ [13]. Room temperature FM has also been found in metal oxides such as $\mathrm{TiO}_{2}, \mathrm{ZnO}, \mathrm{CeO}_{2}$, and $\mathrm{SnO}_{2}$ which is due to oxygen vacancies and quantum confinement effects according to the experimental results [14-18].

However, defects have been recognized to play important roles in inducing RTFM in $\mathrm{ZnO}[19,20]$. This explains the discrepancies often reported in experiments and offers new opportunities to search for the underlying mechanisms. It is very important to highlight that 
RTFM in CdO nanoparticle, was not yet investigated experimentally in details. It is well known that bulk $\mathrm{CdO}$ powder is diamagnetic oxide with a magnetic susceptibility approximately -0.232 $\times 10^{-6}$ cgs at $300 \mathrm{~K}[21]$. Recently, Electronic structure calculations show that the CdO doped by a nonmagnetic $2 p$ light element $(\mathrm{N})$ lead to spin magnetic moment, and the $p$ - $d$ exchange like $p-p$ coupling interaction was suggested to be responsible for the ferromagnetism [22].

In the present work, $\mathrm{CdO}$ powder was prepared by thermal decomposition method in order to discover the possibility of the appearance of RTFM. For that purpose, structural, optical, and magnetic properties of $\mathrm{CdO}$ powder as well as $\mathrm{CdO}$ powder annealed under vacuum and $\mathrm{H}_{2}$ gas were systematically investigated. Additionally, theoretical calculations based on density functional theory were carried out in order to study the principle possibility of the presence of RTFM in CdO powder (nanoparticles). This study might help to discover the main reasons for the creation of RTFM as long as it is origin in DMSs remains a very controversial topic [23].

\section{Results and discussion}

From previous understandings of FM phenomenon, the short range direct exchange magnetic interaction between dopant ions should be responsible for the presence and/or creation of RTFM. However, conditions for such interaction cannot be present within undoped or slightly doped oxide semiconductors. Therefore, there should exist others mechanisms that would help to realize such interactions. In particular, there must be some medium through which that interaction can develop. Thus, one of the most acceptable explanations for the appearance of FM in DMS is the magnetic interaction between dopant TM ions via itinerant carriers like electrons. Such carrier mediated magnetic interaction give rise to an effective FM coupling between TM spins. Another intermediary property that facilitates the exchange coupling between the localized 
dopant magnetic ions could be realized by overlapping of polarons (or bound magnetic polarons BMPs) [24]. In the BMP model, it was established that the major option to obtain higher Curie temperature $\left(\mathrm{T}_{\mathrm{C}}\right)$ is to increase the donor electron density around the magnetic impurities. Some sources attribute creation of RTFM in TCOs to the defects, oxygen vacancies [25-27] metal vacancies $^{15}$ and nano size of the grains of the material. The aim of the present work is to examine for the first time the possibility of appearance of RTFM in undoped CdO powder prepared at the nanoscale then followed by annealing under various atmospheres (air, hydrogen, and vacuum) in order to introduce point defects, which help to shed the light on the origin of RTFM (see Supplemental Material [28]).

XRD patterns of as-prepared and annealed $\mathrm{CdO}$ powders under air, vacuum, and $\mathrm{H}_{2}$ gas are displayed in Fig. 1. It shows that all synthesized powders are polycrystalline in nature with usual cubic $\mathrm{CdO}(\mathrm{Fm}-3 \mathrm{~m})$ structure. X-ray data of structural analysis were collected in Table 1 and indicated that the calculated lattice parameter (by least squares method) for CdO powders are close to the JCPDS card No. 05-0640 [29]. However, annealing under $\mathrm{H}_{2}$ gas or vacuum creates defects and missing atoms, which cause reduction in the unit-cell volume. Thus, the structural bulk compressive microstrain can be expressed by: $\varepsilon_{\text {Bulk }}=-\Delta V_{\text {cell }} / V_{\text {cell }}^{0}$, where $V_{\text {cell }}^{0}$ is the unit-cell volume of the synthesized air-annealed $\mathrm{CdO}$ powder as a reference (see Table 1). We have to mention that by using the above definition of the resultant microstrain $\left(\varepsilon_{B u l k}\right)$, we did not conduct any calculations on its elements like the contribution from the confinement for nanopowder. This compressive strain is caused by a compressive bulk stress $\left(\sigma_{s t}\right)$ that can be estimated with $\sigma_{s t}=\varepsilon_{\text {Bulk }} B$, where $\mathrm{B}$ is the average bulk modulus of $\mathrm{CdO}$, which is about 158 $\mathrm{GPa}[30]$. However, the created stress can produce a slight decrease in the lattice parameter. The creation of point defect vacancies due to the annealing under vacuum and $\mathrm{H}_{2}$ gas might be the 
main responsible for that unit-cell volume strain. Note that more strain is created under vacuum annealing compared to that under annealing under $\mathrm{H}_{2}$ gas. This can be explained as follow: (i) annealing under vacuum mainly creates oxygen vacancies while annealing under $\mathrm{H}_{2}$ gas mainly makes $\mathrm{Cd}$ vacancies, (ii) the ionic radius of $\mathrm{O}^{2-}$ ion $(6$ coordination) is $0.14 \mathrm{~nm}$ while that of $\mathrm{Cd}^{2+}$ is $0.095 \mathrm{~nm}$ [31], and (iii) the creation of $\mathrm{O}^{2-}$ vacancies $\left(\mathrm{V}_{\mathrm{O}}\right)$ produces more volume strain than that of $\mathrm{Cd}^{2+}$ vacancies $\left(\mathrm{V}_{\mathrm{Cd}}\right)$. The strain $(\varepsilon)$ induced in powders due to crystal imperfection and distortion was calculated using the formula [32]: $\varepsilon=\beta_{\mathrm{hkl}} / 4 \tan \theta_{\mathrm{hkl}}$, where $\beta_{\mathrm{hkl}}$ is the half width at half maximum of $(h k l)$ reflection (in rad) and $\theta_{\mathrm{hkl}}$ is the Bragg angle of $(h k l)$ reflection, which was (111) (see Table 1). In general, the lattice strain $(\varepsilon)$ was not greatly influenced by the nature of annealing atmosphere. However, it slightly increases with $\mathrm{H}_{2}$ annealing.

Cross section transmission electron microscope (TEM) images of $\mathrm{CdO}$ powders annealed in air, vacuum, and $\mathrm{H}_{2}$ atmospheres are depicted in Fig. 2. It can be seen that the average particle size of CdO sample (Figure 2a) was in the range of 5-10 $\mathrm{nm}$ range. These particles exhibit are in the crystalline phase but exhibit random shapes. The crystal structure of $\mathrm{CdO}$ sample was found out from the calculated fast-Fourier transform (FFT) of the micrograph which is shown as an insert in Fig. 2a. The spots in the FFT analysis matched with d-spacing of $\mathrm{CdO}$ face-centered cubic (fcc) with rock-salt structure. Hence the TEM analysis corroborated the rock-salt crystal structure of $\mathrm{CdO}$ samples. Additionally the EDS analysis was performed to determine the elemental composition of the samples, as shown in Fig. 3. The spectrum (Fig. 3a) shows that the samples were pure from having any impurities and furthermore the relative elemental composition of $\mathrm{Cd}$ and $\mathrm{O}$ was close 1:1. On the other hand, the $\mathrm{CdO}$-vac sample was $\mathrm{Cd}$ rich (composition Cd:O:: 55:45) while the CdO-H sample was found to be oxygen rich (composition $\mathrm{Cd}: \mathrm{O}$ is 65:35). This indicates the creation of vacancies in CdO-vac sample and $\mathrm{Cd}$ vacancies in 
$\mathrm{CdO}-\mathrm{H}$, hence supports the results from XRD analysis. The higher amount of oxygen in $\mathrm{CdO}-\mathrm{H}$ sample could mean that oxygen is present either in interstitial sites in the bulk of $\mathrm{CdO}$ or it is present at the surface sites making perhaps an oxygen-rich oxide at the surface. Similarly, Figure $3 \mathrm{~b}$ and $3 \mathrm{c}$ contain TEM images of $\mathrm{CdO}-\mathrm{vac}$ and $\mathrm{CdO}-\mathrm{H}$ samples, respectively. In fact no significant difference in their micrographs was noticed as compared to the case of CdO sample. In fact, the particle size also close to $5-10 \mathrm{~nm}$ and the structure was found out to be also fcc rocksalt.

The $\mathrm{CdO}$ samples (annealed in air, annealed in vacuum and annealed under hydrogen) were magnetically characterized. The measured room temperature magnetization curves for $\mathrm{CdO}$ samples annealed in air, annealed in vacuum and hydrogen atmosphere samples are shown in Fig. 4. The treated samples exhibited the similar magnetic ordering, which found to be dominated by a diamagnetic component. However when the diamagnetic component is removed, the $\mathrm{CdO}$ samples exhibited a ferromagnetic behavior with a coercivity around 100 Oe and small saturation magnetization which is observed to be around $\approx 0.0011 \mathrm{emu} / \mathrm{g}$ for the annealed in air and hydrogenated samples. However, for the sample annealed under vacuum, a slightly higher saturation magnetization of $0.0015 \mathrm{emu} / \mathrm{g}$ is observed. The ferromagnetism in the undoped metal oxides like $\mathrm{ZnO}$ and $\mathrm{MgO}$ have been observed in the past and attributed to vacancy defects [3338]. From the measured ferromagnetic ordering for the samples examined, it can be observed that the preparation conditions have little effects on the enhancement of the defects induced ferromagnetic long-range order.

In order to understand the origin of magnetism in $\mathrm{CdO}$ nanoparticles (NP) with oxygen and cadmium vacancies and mimic the samples prepared in the experimental section, we carried out first-principles calculations based on density functional theory [39-42] (see Supplemental 
Material [28]). A $2 \mathrm{~nm}$ diameter, faceted $\mathrm{CdO}$ nanocrystal was modeled from known surface energies of $\mathrm{CdO}$ using the Wulff construction [43]. This construction leads to CdO-NP having 341 atoms, with $177 \mathrm{Cd}$ and $164 \mathrm{O}$ atoms. Then, we generated a $\mathrm{Cd}$ and $\mathrm{O}$ vacancies by removing three $\mathrm{Cd}$ or $\mathrm{O}$ atoms on each side of the surface and center of the CdO NP. This led to a $\mathrm{CdO} \mathrm{NP}$ with $\mathrm{Cd}$ or $\mathrm{O}$ vacancies concentration of $0.88 \%$. Figure 5 shows the calculated total energy in order to compare the structural stability for different vacancy types (Cd or O surface and $\mathrm{Cd}$ or $\mathrm{O}$ center) as well as spin density iso-surfaces generated from vacancies. We can see that the most stable configuration is the CdO-NP with surface O-defects. Note that in all cases the oxygen atoms are negatively polarized (negative magnetic moment) whereas the $\mathrm{Cd}$ atoms are positively polarized. In Cd-defect case, the magnetization is confined to the surface and with short range, whereas for the other case, the polarization is "spread" in the center or all the surfaces of the CdO-NP. Moreover, the spin density iso-surfaces show a clear polarization for all cases. To further illustration the electronic structure and address the origin of the ferromagnetism resulting from $\mathrm{Cd}$ and $\mathrm{O}$ vacancies, we calculated the spin resolved total densities of states (DOS), as plotted in Figure 6. This shows that $\mathrm{Cd}$ and $\mathrm{O}$ vacancies induce spin polarization of the top of the valence band. We note that there is a significant change in the spin-up and spindown total DOS at the Fermi level compared to pure CdO. To understand this change, we calculated the local DOS of $\mathrm{Cd}$ and $\mathrm{O}$ atoms situated near the created vacancies, as shown in Fig.2. Interestingly, they indicate that the LDOS of (a), (b) and (c) configurations show different behavior compared to (d). In the (a), (b) and (c) cases, the LDOS shows a strong hybridization between $\mathrm{Cd} d$ and $2 p$ orbitals near the Fermi level. While in the case of (d), the O $2 p$ orbital interacts slightly with the Cd $4 d$ orbital, and O $2 p$ states appear at the Fermi level. 
From the DFT calculations, it has been predicted that for a $0.88 \%$ vacancy defects of $\mathrm{Cd}$ ions in the crystal structure give rise to a magnetic moment of $0.8 \mu_{\mathrm{B}}$ per vacancy as in case of $\mathrm{ZnO}$ with $\mathrm{Zn}$ vacancies [12]. For the examined samples, the number of $\mathrm{Cd}$ ions that correspond to a $0.88 \%$ vacancy defects is equal to $4.12 \times 10^{19}$ vacancies/g. Assuming that all the vacancy defects possess a magnetic moment and by considering that all these magnetic moments contribute to the long range ferromagnetic order, then a magnetic moment of $0.003 \mu_{\mathrm{B}}$ per vacancy is estimated using the observed saturation value for the ferromagnetic component $\left(\mathrm{M}_{\mathrm{s}}=\mathrm{N}_{\text {vacancy }} \times \mathrm{m}\right)$. Accordingly, this estimated value for the magnetic moment is much lower than the predicted value $\left(0.8 \mu_{\mathrm{B}}\right.$ per vacancy) which is expected since not all the existing vacancies inducing magnetic moment are necessarily coupled and contributed to the long-range ferromagnetic order. It is important to note that uncoupled magnetic moments usually give rise to a paramagnetic component that will be masked by the diamagnetic component since both components varies linearly in the range of the used magnetic field. According to the above experimental estimation of the magnetic moment per $\mathrm{Cd}$ vacancy from $\mathrm{M}-\mathrm{H}$ curves, as summarized in Table 2 , we observe that only $[(0.003-0.8 / 0.8) \times 100=99.625 \%]$ a small proportion of about $0.375 \%$ participate in the observed FM phase from the supposed $0.88 \%$.

\section{Summary}

The effect of post annealing under vacuum and hydrogen atmosphere of $\mathrm{CdO}$ powder was investigated. X-ray diffraction analysis confirms the formation of single $\mathrm{CdO}$ phase with no significant effect due to the post annealing. TEM analysis has demonstrated the formation of nanoparticles in the range of 5-10 nm. Magnetic measurements reveal the appearance of ferromagnetic phase with a dominant diamagnetic component for all powders, with slight enhancement of the saturation magnetization due to post annealing, by $36 \%$ and $9 \%$ for vacuum 
and hydrogen treated samples respectively. Theoretically, we have applied ab initio calculations in order to describe the electronic structure and magnetic properties of $\mathrm{CdO}$ nanoparticles with $0.88 \%$ of $\mathrm{Cd}$ vacancies. Interestingly, we find that these vacancies induce spin-polarization of $\mathrm{O}$ $2 p$ and $\mathrm{Cd} 4 \mathrm{~d}$ orbitals near the Fermi level, generating ferromagnetic coupled vacancies with a magnetic moment of $0.8 \mu_{\mathrm{B}}$ per vacancy. 


\section{References}

[1] B. J. Lewis, D. C. Paine, Mater. Res. Soc. Bull. 25, 22-27 (2000).

[2] M. Yan, M. Lane, C. R. Kannewurf, R. P. H. Chang, Appl. Phys. Lett. 78, 02342- 02345 (2001).

[3] D. M. Carballeda-Galicia, R. Castanedo-Perez, O. Jimenez-Sandoval, S. Jimenez-Sandoval, G. Torres-Delgado, C. I. Zuniga-Romero, Thin Solid Films 371, 105-108 (2000).

[4] O. Gomez Daza, A. Arias-Carbajal Readigos, J. Campos, M. T. S. Nair, P. K. Nair, Modern Phys. Lett. B 17, 609-939 (2001).

[5] T. Dietl, H. Ohno, F. Matsukura, J. Cibert, D. Ferrand, Zener Science 287, 1019-1022 (2000).

[6] M. Venkatesan, C. B. Fitzgerald, J. M. D. Coey. Nature (London) 430, 630 (2004).

[7] J. M. D Coey. Solid State Sci. 7, 660-667 (2005).

[8] C. Das Pemmaraju, S. Sanvito, Phys. Rev. Lett. 94, 217205-4 (2005).

[9] H. Peng, J. Li, S.-S. Li, J.-B. Xia Phys. Rev. B 79, 092411 (2009).

[10] A. Janotti, J. B. Varley, P. Rinke, N. Umezawa, G. Kresse, C. G. Van de Walle, Phys. Rev. B 81, 085212 (2010).

[11] H. Peng, H. J. Xiang, S.-H. Wei, S.-S. Li,; J.-B. Xia, J. Li, Phys. Rev. Lett. 102, 017201 (2009).

[12] M. B. Kanoun, S. Goumri-Said, U. Schwingenschlögl, A. Manchon, Chem. Phys. Lett. 532 96-99 (2012).

[13] V. Fernandes, R. J. O. Mossanek, P. Schio, J. J. Klein, A. J. A. de Oliveira, W. A. Ortiz, N. Mattoso, J. Varalda, W. H. Schreiner, M. Abbate, D. H. Mosca, Phys. Rev. B 80, 035202 (2009). [14] C. Guglieri, E. Céspedes, A. Espinosa, M. Á. Laguna-Marco, N. Carmona, Y. Takeda, T.

Okane, T. Nakamura, M. García-Hernández, M. Ángel García, J. Chaboy, Adv. Funct. Mater. 24, 2094 (2014).

[15] S. B. Ogale Adv. Mater. 22, 3125-3155 (2010)

[16] S. Zhang, C. I. Pelligra, G. Keskar, P. W. Majewski, F. Ren, L. D. Pfefferle, C. O. Osuji, ACS Nano, 5, 10, 8357 (2011)

[17] N. H. Hong, J. Sakai, N. Poirot, V. Brizé, Phys. Rev. B 73, 132404 (2006).

[18] A. Sundaresan, R. Bhargavi, N. Rangarajan, U. Siddesh, U.; C. N. R. Rao, Phys. Rev. B 74, 161306(R) (2006). 
[19] J.B. Yi, C.C. Lim, G.Z. Xing, H.M. Fan, L.H. Van, S.L. Huang, K.S. Yang, X.L. Huang, X.B. Qin, B.Y. Wang, T. Wu, L. Wang, et al., Phys. Rev. Lett. 104, 137201 (2010).

[20] G. Xing, D. Wang, J. Yi, L. Yang, M. Gao, M. He, J. Yang, J. Ding, T. Chien Sum, T. Wu, Appl. Phys. Lett. 96, 112511 (2010).

[21] R. Chandiramouli, B.G. Jeyaprakash, Solid State Sci., 16, 102-110 (2013).

[22] C.-W. Zhang, P.-J. Wang, Y. Su, Phys. Lett. A 374, 1889-1892 (2010).

[23] Y. F. Chen, Q.G. Song, H.Y. Yan, Comp. Theor. Chem. 983, 65 (2012).

[24] A. Kaminski, S.D. Sarma, Phys. Rev. Lett. 88247202 (2002).

[25] A. Hakeem, J. Mag. Mag. Mater. 322, 709 (2010).

[26] A. F. Kohan, G. Ceder, D. Morgan, C. G. Van de Walle, Phys. Rev. B 61, 15019-15027 (2000).

[27] B. Choudhury, A. Choudhurym, A. K. M. Maidul Islam, P. Alagarsmy, M. Mukhherjee, J. Mag. Mag. Mater 323, 440-446 (2011).

[28] See Supplemental Material for technical descriptions of experiment and methods of calculations. X-ray fluorescence of synthesised $\mathrm{CdO}$ powder is also represented.

[29] Powder Diffraction File, Joint Committee for Powder Diffraction Studies (JCPDS) file No. 05-0640.

[30] J. Zhang, Phys Chem Minerals 26, 644-648 (1999)

[31] R. D. Shannon, Acta Crystallogr. A 32, 751-767 (1976).

[32] V.D. Mote, Y. Purushotham, B.N. Dole, J. Theo. Appl. Phys. 6:6 (2012)

[33] M. Kapilashrami, J. Xu, K. V. Rao, L. Belova, Proc. Appl Ceram. 4, 225 (2010).

[34] J. Hu, Z. Zhang, Z. Zho, H. Qin, M. Jiang, Appl. Phys. Lett. 93, 192503 (2008).

[35] T.-L. Phan, Y. D. Zhang, D. S. Yang, N. X. Nghia, T. D. Thanh, S. C. Yu, Appl. Phys. Lett. 102, 072408 (2013).

[36] J. Zhang, D. Gao, M. Si, Z. Zhu, G. Yang, Z. Shi, D. Xue, J. Mater. Chem. C, 1, 6216 (2013).

[37] S. Azzaza, M. El-Hilo, S. Narayanan, J. Judith Vijaya, N. Mamouni, A. Benyoussef, A. El Kenz, M. Bououdina, Mater. Chem. Phys. 143, 1500 (2014).

[38] B. Małecka, J. Therm. Anal. Calorim. 78, 535 (2004).

[39] J. M. Soler, E. Artacho, J. D. Gale, A. Garcia, J. Junquera, P. Ordejon, D. Sanchez-Portal, J. Phys. Condens. Matter. 14, 2745 (2002). 
[40] J. Junquera, O. Paz, D. Sanchez-Portaland, E. Artacho, Phys. Rev. B 64, 235111 (2001).

[41] N. Troullierand, J. L. Martins, Phys. Rev. B 43, 1993 (1991).

[42] J. P. Perdew, K. Burke, M. Ernzerhof, Phys. Rev. Lett. 77, 3865 (1996).

[43] E. Ringe, R.P. Van Duyne, L.D. Marks, Nano. Lett. 11, 3399-3403 (2011). 
Table 1: Lattice parameter $(a)$, mean crystallite size (CS), and created bulk strain $\left(\varepsilon_{\text {Bulk }}\right)$ for the prepared powders.

\begin{tabular}{cccc}
\hline Preparation Condition & $\boldsymbol{a}(\mathbf{n m})$ & $\mathbf{C S}(\mathbf{n m})$ & $\boldsymbol{\varepsilon}_{\text {Bulk }}(\boldsymbol{\%})$ \\
\hline CdO-air & 4.69981 & 44.8 & ref \\
CdO-Vac & 4.68194 & 46.4 & -1.13 \\
CdO-H & 4.68774 & 39.7 & -0.77 \\
\hline
\end{tabular}

Table 2: Obtained magnetic parameters for the examined $\mathrm{CdO}$ samples at different preparation conditions.

\begin{tabular}{cccc}
\hline Preparation Condition & $\chi_{\mathbf{d}}(\mathbf{e m u} /$ Oe.g $)$ & $\mathbf{M}_{\mathbf{s}}(\mathbf{e m u} / \mathbf{g})$ & $\mathbf{H}_{\mathbf{c}}(\mathbf{O e})$ \\
\hline CdO-air & $3.575 \mathrm{e}^{-7}$ & 0.001 & \\
CdO-Vac & $3.2 \mathrm{e}^{-7}$ & 0.00145 & 100 \\
CdO-H & $3.47 \mathrm{e}^{-7}$ & 0.00116 & 95 \\
\hline
\end{tabular}




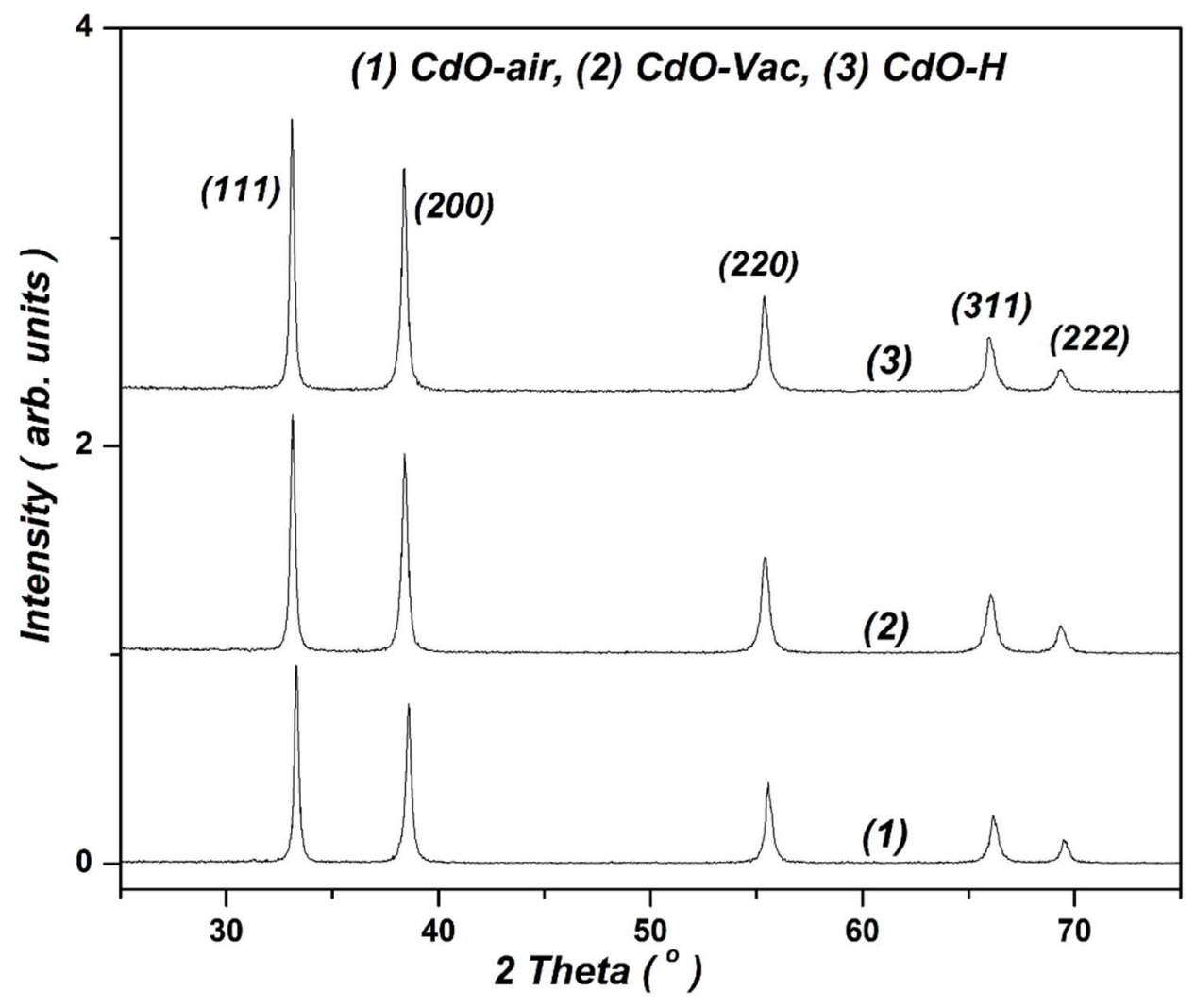

Figure 1. XRD patterns of prepared $\mathrm{CdO}$ powders annealed in air, vacuum, and $\mathrm{H}_{2}$ atmospheres.

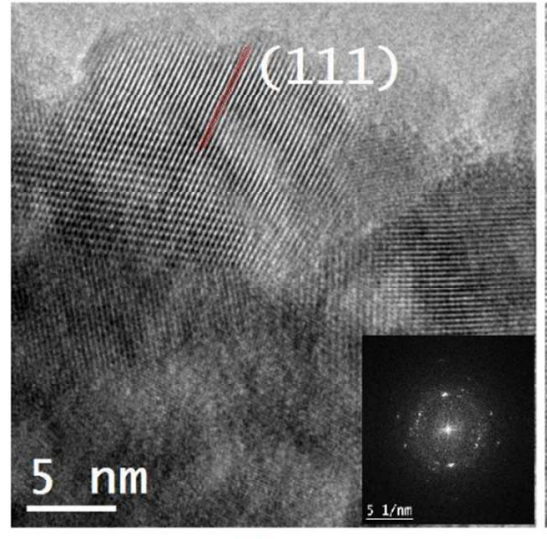

(a)

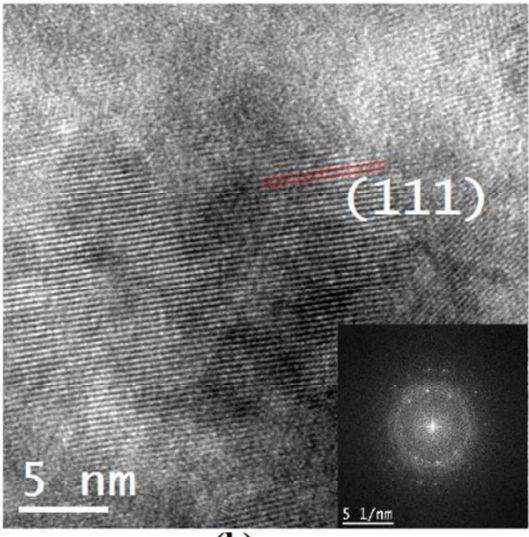

(b)

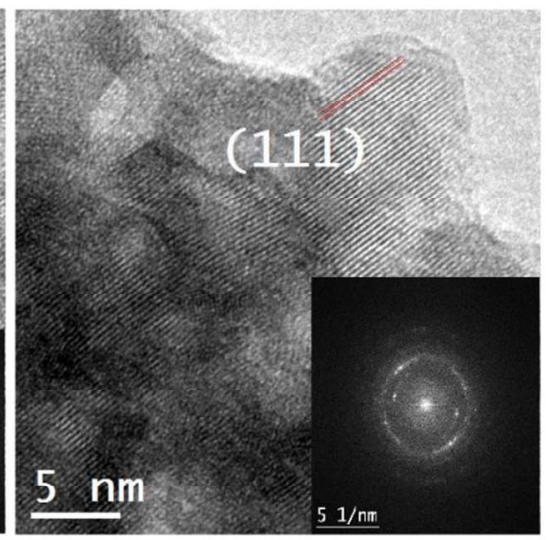

(c)

Figure 2. TEM analysis of $\mathrm{CdO}$ powders annealed in air, vacuum, and $\mathrm{H}_{2}$ atmospheres. 


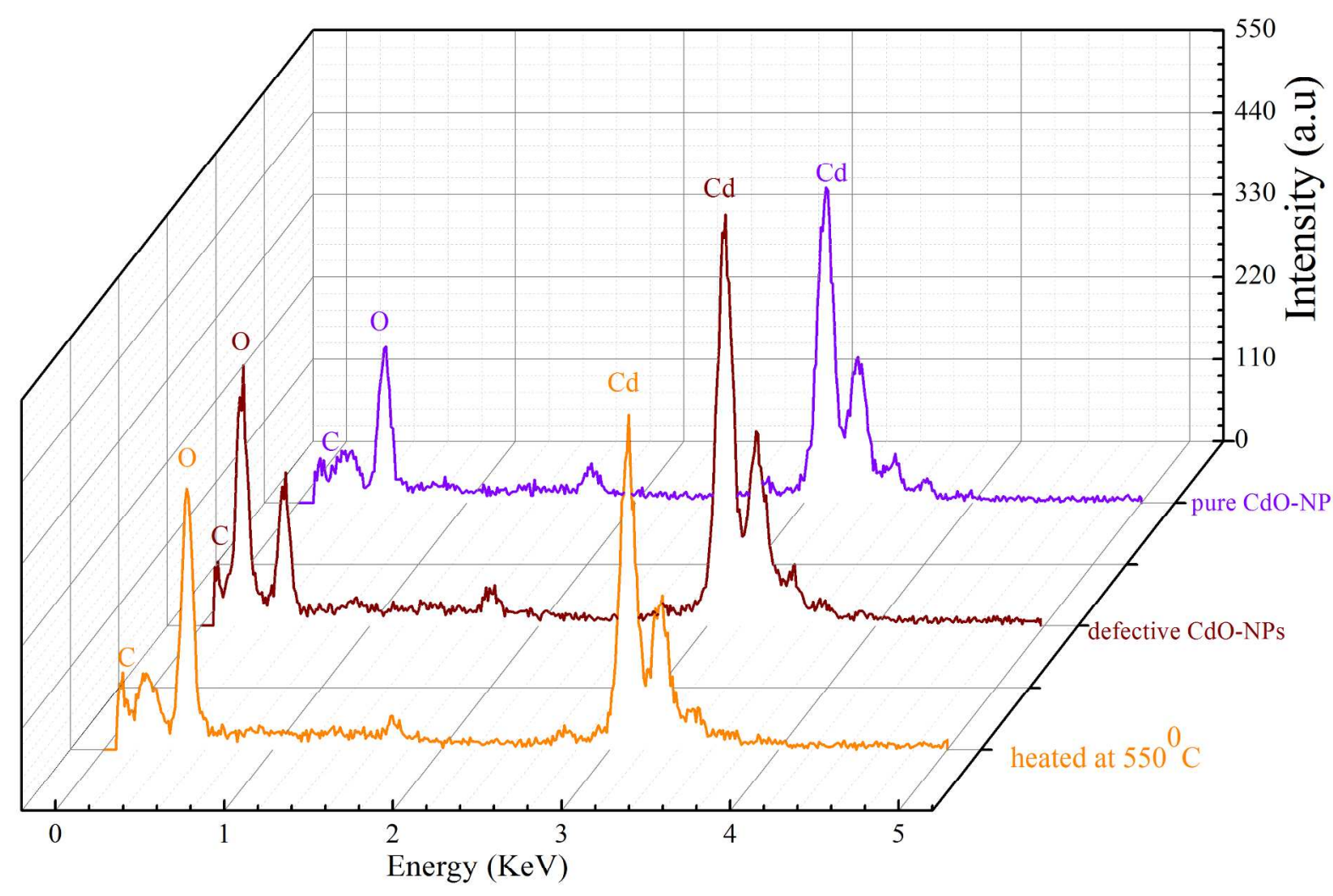

Figure 3. EDS spectra of $\mathrm{CdO}$ powders annealed in air, vacuum, and $\mathrm{H}_{2}$ atmospheres.
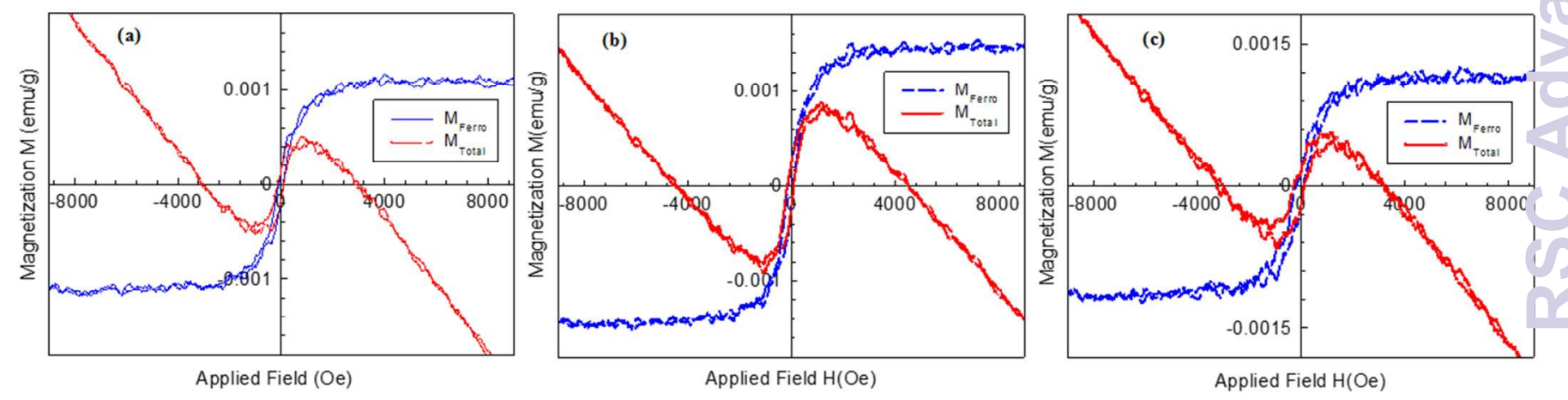

Figure 4. The measured room temperature magnetisation curves for: (a) pure as prepared $\mathrm{CdO}$; (b) $\mathrm{CdO}$ annealed in vacuum and (c) $\mathrm{CdO}$ annealed under Hydrogen. 


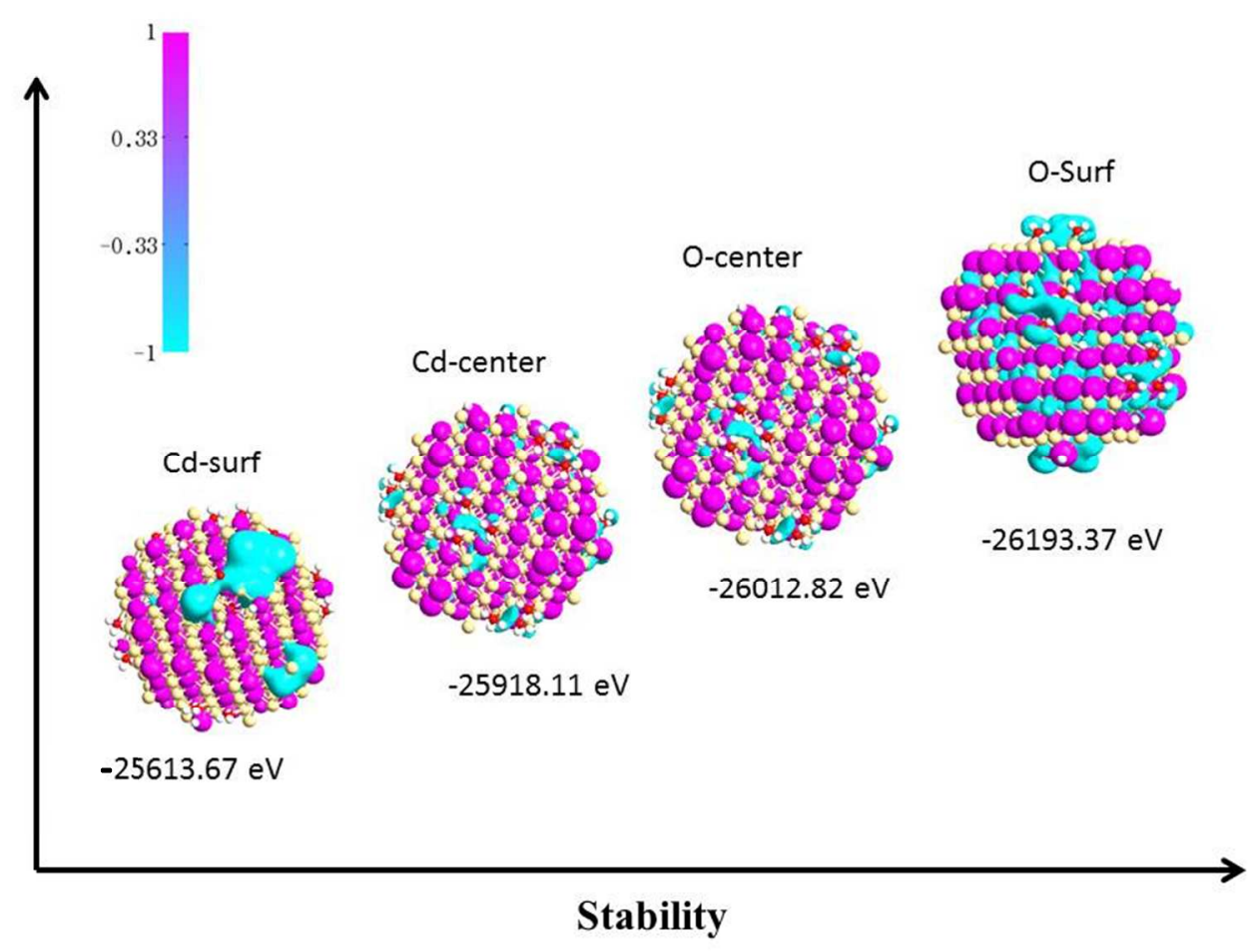

Figure 5. Spin-density isosurfaces and total energies versus the growing energetic stability of $\mathrm{CdO}$ (with $\mathrm{Cd}$ and $\mathrm{O}$ at surface (surf) and center). 

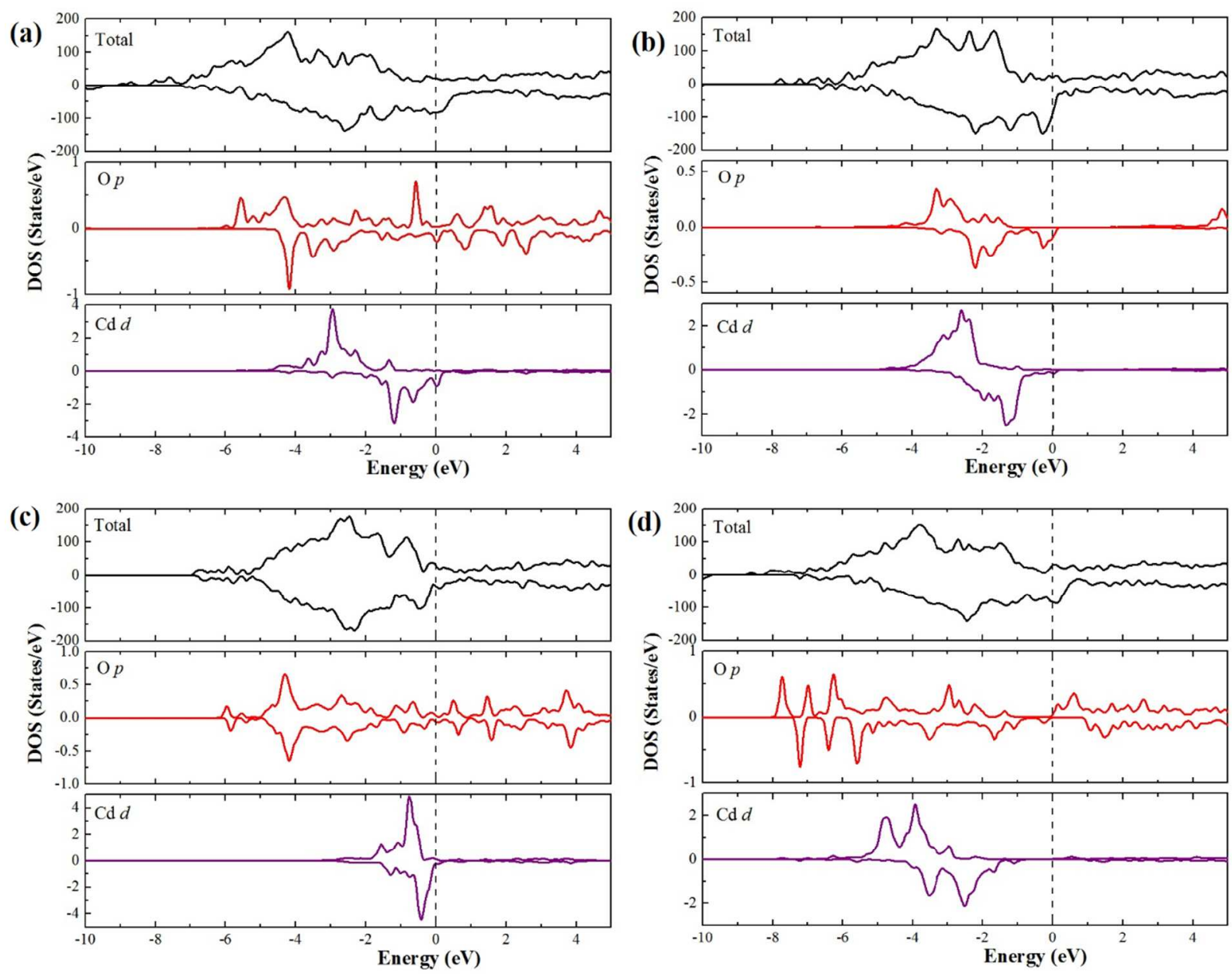

Figure 6. Total and local densities of states of Cd-d and O-p states. (a) Cd-surf, (b) Cd-center, (c) O-surf and (d) O-center cases. 


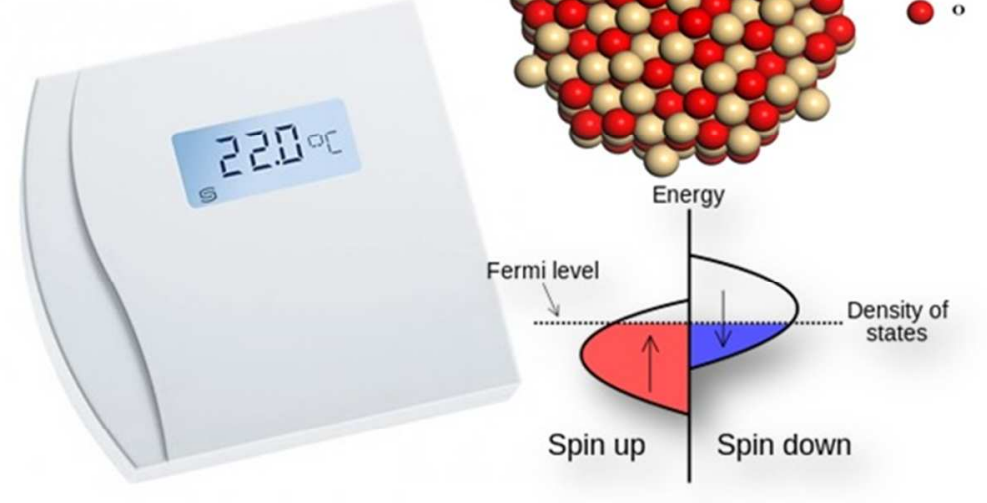

